\title{
Universitarios mexicanos: lo mejor y lo peor de la pandemia de COVID-19
}

\author{
Andrés Melchor Audirac, Andrea Guadalupe Hernández Zúñiga \\ y Juan José Sánchez Sosa
}

\begin{abstract}
Resumen
La pandemia de la covid-19 ha provocado cambios radicales en las diversas actividades humanas, entre las más afectadas: la educación. El objetivo del presente estudio fue explorar las percepciones sobre lo mejor y lo peor que ha causado la pandemia de la covid-19 en los estudiantes de la Facultad de Psicología de la unam. Para lograrlo, se realizó una investigación cualitativa por medio de un sondeo de opinión exploratorio. Se obtuvo una muestra de 80 alumnos de licenciatura, maestría y doctorado, con un total de 271 respuestas. La cancelación de planes personales, académicos y profesionales, así como emociones negativas como enojo, irritabilidad y tristeza, al igual que un aumento de estrés, ansiedad y depresión, fueron los temas más frecuentes reportados por los estudiantes como lo peor que trajo la pandemia. Por otro lado, el estar más tiempo con la familia y seres queridos, y un aumento en conductas de autocuidado como mejor alimentación, sueño y ejercicio se reflejaron como lo mejor de este mismo fenómeno. Es indispensable conocer las experiencias de los universitarios ante cambios radicales que atraviesan individualmente en las esferas de su vida durante esta crisis de salud pública, ya que tiene un impacto en su educación.
\end{abstract}

Palabras clave: COVID-19, Estudiantes Universitarios, Experiencias, Sondeo de Opinión, Distanciamiento Social.

\section{Mexican University Students: The Best and Worst of the COVID-19 Pandemic}

\begin{abstract}
The covID-19 pandemic has caused radical changes in diverse human activities. Among the most affected: education. The objective of the present study is to explore the perceptions of the best and the worst that the covid-19 pandemic has caused in college students of the Faculty of Psychology at unAm. To achieve this, a qualitative research was carried out through an exploratory opinion poll. The sample was composed of 80 undergraduates and graduate students, collecting a total of 271 responses.

The postponement of personal, academic, and professional plans, the onset of negative emotions such as anger, irritability, and sadness, as well as an increase of stress, anxiety, and depression, were the most frequent topics reported by students as the worst that the pandemic brought. On the other hand, spending more time with their families, and a rise of self-care behaviors, such as better nutrition, sleep, and physical exercise were reported as the best experiences regarding the pandemic. It is essential to know the struggles of college students to cope with radical changes that they go through in the diverse spheres of their life, during this public health crisis since it has an impact on their education.
\end{abstract}

Keywords: COVID-19, University Students, Experiences, Opinion Poll, Social Distancing

Recepción: 18/01/2021. Aprobación: 23/03/2021. Dol: http://doi.org/10.22201/cuaieed.16076079e.2021.22.3.12 
Licenciado en Psicología por la Facultad de Psicología de la Universidad Nacional Autónoma de México. Durante sus estudios de pregrado realizó una estancia académica en la Universidad de Groningen en Los Países Bajos. Por más de un año fue asistente de investigación de SNI II del conAcYT. Actualmente forma parte de un proyecto de investigación en el Instituto Nacional de Psiquiatría "Ramón de la Fuente Muñiz" en colaboración con la Escuela de Medicina de Harvard y financiado por el Instituto Nacional de Salud Mental de Estados Unidos.

Andrea Guadalupe Hernández Zúñiga

aghz1296@gmail.com orcid.org/0000-0002-4254-6023

Pasante de psicología de la Facultad de Psicología de la Universidad Nacional Autónoma de México. Durante sus estudios de pregrado realizó una estancia académica en la Universidad de Antioquia en Colombia. Cuenta con experiencia de prácticas profesionales en el área de Cuidados Paliativos, atención a personas que viven con VIH y evaluación psicológica con niños y adolescentes. Actualmente funge como asistente de investigación de SNI III del conACrT.

Juan José Sánchez Sosa

jujosaso@gmail.com orcid.org/0000-0001-7676-5569

El Dr. Sánchez Sosa es Psicólogo por la unam. Obtuvo la Maestría y el Doctorado en Psicología y Desarrollo Humano en la Universidad de Kansas, EUA y estudios de actualización en la Clínica Menninger y el programa conjunto de educación continua del Hospital General de Massachusetts y la Escuela de Medicina de Harvard. Es profesor de Psicología de la unAM, en el área de psicología de la salud, ha dirigido más de 130 tesis de doctorado, maestría y licenciatura, y ha sido sinodal en exámenes de doctorado por invitación en universidades de Estados Unidos, Holanda, Suiza y España.

Entre otras distinciones, El Dr. Sánchez-Sosa ha recibido el Doctorado Honoris Causa por la Universidad de Ottawa, Canadá; el Premio Universidad Nacional en Ciencias Sociales, el nivel ॥I como Investigador Nacional (sNI), la Residencia Fulbright en la Universidad de California, el premio "Outstanding International Psychologist Award" de la American Psychological Association y es Profesor Emérito de la unAM. 


\section{Introducción}

A inicios del 2020, en China surgió un nuevo virus conocido como sARs-CoV-2, que ha causado afectaciones en el sistema respiratorio de las personas infectadas. En poco tiempo este virus se esparció por todo el mundo, convirtiéndose en la pandemia más importante de nuestra era. El método más eficaz para combatir el incremento exponencial de personas infectadas fue el aislamiento social o cuarentena (oms, 2020). Así que, para el 1 de abril de 2020, más de 3 mil millones de personas en 80 países estaban recluidas en sus hogares para frenar la propagación del virus (UNESCO, 2020). Con esto, diversas actividades humanas cambiaron radicalmente, entre las más afectadas: la educación.

Con base en la encuesta realizada por la Asociación Internacional de Educación Superior en abril de 2020 (Marinoni, Van't Land y Jensen, 2020), los recintos universitarios alrededor del mundo cerraron debido a la normatividad de distanciamiento social, lo que ha afectado a más de 1.5 mil millones de estudiantes en más de 182 naciones. En el continente americano, 72\% de las universidades tuvieron que adaptar su enseñanza presencial a una en línea, en $22 \%$ la educación se encontraba suspendida y en 3\% de las universidades la educación universitaria se canceló.

Debido a ello, surgió la urgencia de adaptar el uso de las nuevas tecnologías de la información y comunicación (TIC) como las herramientas principales para continuar la educación universitaria, provocando así lo que se conoce ahora como la Crisis de la Digitalización de la Educación (Sanz, Sáinz y Capilla, 2020). Dicha crisis no sólo originó un cambio inmediato de las técnicas pedagógicas de enseñanza presencial a una virtual, sino que también acentuó las brechas socioeconómicas entre los estudiantes universitarios, ya que no todos cuentan con la infraestructura o habilidades necesarias para las clases en línea (computadora personal, acceso internet, habilidades en el uso de TIc, etcétera).

No obstante, el escenario actual no sólo es oscuro. La adaptación a una nueva modalidad de enseñanza también permite vislumbrar formas más flexibles para la impartición de clases asincrónicas y en línea, que en un futuro pueden ser implementadas en los planes de estudios en las universidades.

Besser, Fletty Zeigler-Hill (2020) estudiaron el impacto de este cambio brusco y repentino al formato pedagógico en línea en la educación universitaria, debido a la pandemia por la covid-19 y el estado psicológico de los estudiantes universitarios. Dicha investigación brindó hallazgos muy interesantes. Entre los más relevantes, se encontró que el cambio a la modalidad en línea generó, en los universitarios, un incremento significativo de estados de ánimo negativos (ansiedad, frustración, impotencia y aburrimiento). También se observaron niveles elevados de estrés y soledad, aunados a un decremento notorio de concentración, motivación y aprendizaje. Algo sumamente relevante y novedoso en este estudio fue que se incluyó el factor de la adaptabilidad como variable mediadora de efectos negativos 
y positivos en las experiencias del aprendizaje en línea. Los universitarios con alta capacidad de adaptación mostraron ser los que reportaron menos emociones negativas y estrés asociadas a este cambio en la modalidad de sus clases.

Con respecto a cómo los estudiantes universitarios evaluaron la transición forzada de clases presenciales a clases en línea, Garris y Fleck (2020) encontraron que en una universidad de Estados Unidos sus estudiantes evaluaron negativamente el semestre en el cual se hizo dicho cambio. En su mayoría, el alumnado calificó sus cursos como menos interesantes, menos agradables y que incitaban en menor medida al aprendizaje significativo. Estas opiniones tuvieron un impacto negativo en la evaluación de la calidad de enseñanza universitaria.

Dicha evaluación negativa está influenciada por el contexto de la pandemia. Como Cao et al. (2020) exponen en su estudio con estudiantes universitarios chinos, las variables que más afectan la percepción de la calidad de enseñanza son el estrés y la ansiedad. Estas emociones negativas surgieron a raíz del contagio de familiares o del miedo al propio contagio. No es difícil concluir que la incertidumbre ocasionada por la crisis sanitaria tiene un gran efecto negativo en las demás esferas de la vida de un universitario (física, psicológica, académica, económica y social). Dzokoto, Hicks y Miller (2007) hallaron que el estrés causado por conflictos familiares, problemas económicos y un exceso en la carga de trabajo académico son un buen predictor para la depresión en los universitarios.

Bajo este contexto, resulta indispensable conocer la situación de la comunidad universitaria mexicana ante la actual crisis de salud pública internacional. Es importante entender las barreras percibidas ante esta nueva normalidad de la educación, así como los aspectos positivos que han adquirido en estos meses en aislamiento.

\section{Objetivo}

Explorar las percepciones sobre lo bueno y lo malo de la pandemia de la covi-19 por parte de estudiantes de la Facultad de Psicología de la Universidad Nacional Autónoma de México, en sus tres niveles educativos: licenciatura, maestría y doctorado.

\section{Método}

Se realizó un estudio cualitativo por medio de un sondeo de opinión exploratorio sobre las percepciones de lo mejor y lo peor de la pandemia por la covid-19. Se contó con la participación voluntaria de 80 alumnos, 53 mujeres (66.3\%) y 27 hombres (33.7\%) de licenciatura, maestría y doctorado. Se invitó a participar en el sondeo de opinión al término de las clases impartidas por el Dr. Juan José Sánchez Sosa. Se les envió por correo dicha encuesta a todos los alumnos inscritos a los cursos. La pregunta estímulo fue: "Por favor, teclea en un par de renglones, uno con lo más negativo que te ha ocasionado la cuarentena y otro con 10 más positivo". Se recopilaron las respuestas de los alumnos entre el 14 de septiembre al 6 de octubre de 2020. 
Tabla 1. Distribución de la muestra por sexo y por nivel de estudio

Tabla 2. Categorías temáticas: lo más positivo que te ha pasado por la pandemia de la covid-19

\section{Procedimiento}

Se recolectaron 271 respuestas en total, por correo electrónico, de los estudiantes que decidieron contestar a la pregunta estímulo. Se transcribieron todas a un archivo de procesador de textos. La distribución de la muestra por nivel de estudio de los 80 participantes fue: 42 de licenciatura, 29 de maestría y 9 de doctorado. La distribución de la muestra por nivel de estudio y por sexo se ilustra en la tabla 1.

\begin{tabular}{|l|c|c|c|}
\hline \multicolumn{1}{|c|}{ Nivel de Estudio } & Mujeres & Hombres & Total \\
\hline Licenciatura & 32 & 10 & 42 \\
\hline Maestría & 17 & 12 & 29 \\
\hline Doctorado & 4 & 5 & 9 \\
\hline
\end{tabular}

Se realizó una lectura minuciosa de sus experiencias, buscando patrones o ideas que estuvieran relacionados entre los participantes. Posteriormente, se desarrolló un sistema de codificación para las percepciones que tuvieran una temática en común. Para el análisis temático, dos investigadores del presente estudio propusieron clasificaciones para agrupar las respuestas de los participantes en categorías temáticas con base en su contenido. La selección de las respuestas en cada categoría temática se hizo de manera independiente por los investigadores (Corbin y Strauss, 2015). Para tener una alta confiabilidad, se realizó un análisis de concordancia de categorización (Dey, 2007). Se obtuvo un porcentaje de acuerdo de 92.26\%, con sólo 21 respuestas que difirieron de la categorización entre investigadores. En estas últimas hubo un acuerdo en el equipo de investigación y se reasignaron a categorías con base en su contenido.

En la tabla 2, se muestra la clasificación temática de las experiencias más positivas suscitadas por la pandemia de la covid-19. Se presentan el número de participantes incluidos en el estudio divididos por sexo, así como la frecuencia total de cada una de las opiniones por categoría.

\begin{tabular}{|l|c|c|c|}
\hline \multicolumn{1}{|c|}{ Categorías temáticas } & Hombres & Mujeres & Total \\
\hline Más tiempo con familia, pareja y amistades & 13 & 29 & 42 \\
\hline Mayor autocuidado & 7 & 24 & 31 \\
\hline Aprender nuevas cosas & 10 & 10 & 20 \\
\hline Ahorro económico y de tiempo & 6 & 6 & 12 \\
\hline Mejor organización de tiempo & 4 & 8 & 12 \\
\hline Cursos y recursos en línea & 2 & 5 & 7 \\
\hline Nuevas oportunidades laborales & 3 & 4 & 7 \\
\hline Más tolerancia a la incertidumbre o frustración & 3 & 2 & 5 \\
\hline Valorar lo que se tiene & 0 & 4 & 4 \\
\hline Descansar más & 0 & 4 & 4 \\
\hline Total de respuestas recolectadas & 48 & 96 & 144 \\
\hline
\end{tabular}


Tabla 3. Categorías temáticas: lo más negativo que te ha pasado por la pandemia de la covid-19

En la tabla 3, se muestra la clasificación temática de las experiencias más negativas suscitadas por la pandemia de covid-19. En la tabla se presentan el número de participantes incluidos en el estudio divididos por sexo, así como la frecuencia total de cada una de las opiniones por categoría.

\begin{tabular}{|l|c|c|c|}
\hline \multicolumn{1}{|c|}{ Categorías temáticas } & Hombres & Mujeres & Total \\
\hline $\begin{array}{l}\text { Cancelación de planes personales, académicos y } \\
\text { profesionales }\end{array}$ & 8 & 18 & 26 \\
\hline Problemas emocionales & 8 & 14 & 22 \\
\hline Sensación de soledad y aislamiento & 5 & 13 & 18 \\
\hline Dificultades para adaptarse a la cuarentena & 8 & 8 & 16 \\
\hline Conflictos interpersonales con familiares y pareja & 2 & 7 & 9 \\
\hline Problemas económicos o pérdida de empleo & 1 & 8 & 9 \\
\hline Fallecimiento de seres queridos & 4 & 4 & 8 \\
\hline Sensación de incertidumbre por el futuro & 0 & 6 & 6 \\
\hline No poder ver a mis seres queridos & 3 & 2 & 5 \\
\hline Miedo al contagio & 1 & 3 & 4 \\
\hline Contagio por CoviD-19 & 3 & 1 & 4 \\
\hline Total de respuestas recolectadas & 43 & 84 & 127 \\
\hline
\end{tabular}

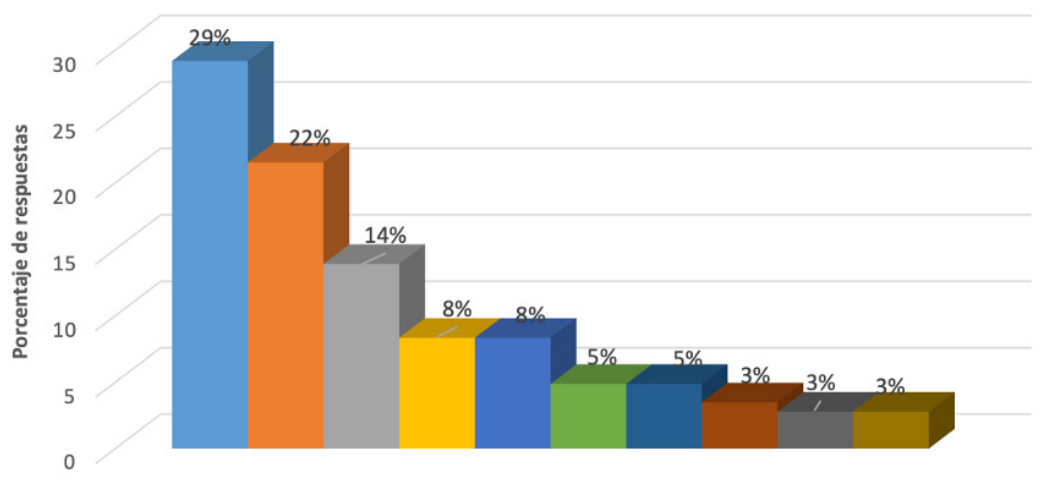

Una vez agrupadas todas las experiencias reportadas por los alumnos encuestados, se contabilizó la frecuencia de experiencias de los participantes en cada una de las categorías temáticas, para determinar el número total de respuestas por cada categoría y obtener su respectivo porcentaje.

\section{Resultados}

\section{Experiencias positivas dentro de la pandemia por Covid-19}

- Mayor autocuidado
Aprender nuevas cosas
- Mejoro económico y en tiemponización de tiempo
- Cursos y recursos en línea
- Nuevas oportunidades laborales
- Más tolerancia a la incertidumbre o frustración
- Valorar lo que se tiene
Descansar más

Gráfica 1. Porcentaje de respuestas de "Lo más positivo de la pandemia de covid-19".

familias, con 29\% de experiencias reportadas de la muestra del presente estudio:

La gráfica 1 ilustra los porcentajes de opinión acerca de las mejores experiencias que se suscitaron a partir de la pandemia por COVID-19.

Dentro de las mejores experiencias que ocasionó la pandemia por la covid-19, se reportó con mayor frecuencia por los participantes el pasar más tiempo con sus

"Comparto más tiempo con mi familia y mis mascotas, lo que contribuye a un sentimiento de bienestar". 
"Pasar más tiempo con mi familia porque normalmente no vivo con ellos entre semana por la lejanía entre mi casa y la facultad".

22\% de las opiniones de los alumnos encuestados mencionó haber mejorado su autocuidado. En esta categoría se describieron varias conductas relacionadas con el autocuidado de la persona. De acuerdo con la definición de la oms (2019), el autocuidado es el comportamiento del individuo que promueve y preserva un estilo de vida saludable o está enfocado en la prevención de la enfermedad. Es un concepto amplio que abarca desde la buena higiene, nutrición, estilos de vida saludables (ejercicio, actividades recreativas, etcétera), factores ambientales y económicos.

Existieron diversas opiniones acerca de una mejor nutrición a causa de estar más tiempo en casa y no tener que alimentarse fuera de ella debido a las largas jornadas en la universidad. De igual manera, los estudiantes encuestados reportaron haber incorporado el ejercicio físico en sus rutinas cotidianas, dado que cuentan con más tiempo para realizarlo. El cuidado de la salud mental también se incluyó dentro de esta categoría, hubo comentarios referentes al tratamiento psicológico por medio de telepsicología, al igual que el experimentar emociones y pensamientos de sí mismos.

Es importante hacer notar que en esta categoría hubo una diferencia entre el número de opiniones con respecto al sexo de los participantes. Como se ilustra en la gráfica 2, el autocuidado se trató de experiencias positivas para 25\% de las mujeres, mientras que lo fue para $14.3 \%$ de hombres en el estudio. También es relevante recalcar que la muestra del presente estudio no tuvo una paridad entre el número de participantes hombres y mujeres, por lo que esta diferencia puede deberse a ello. Dentro de las opiniones más representativas en este rubro se encuentran:

"Mejora de calidad de sueño y hábitos alimenticios".

"Aprender a organizar mis tiempos y prestar atención a mis emociones y pensamientos".

"Poder destinar más tiempo a áreas de mi vida que había mantenido descuidadas como salud (alimentación sana, actividad física y recreación)".

Gráfica 2. Porcentajes de opiniones divididas por sexo en "Lo más positivo de la pandemia de covid-19". Nota: es importante señalar que no hubo una distribución homogénea entre el número de participantes por sexo: $66.3 \%$ mujeres y $33.7 \%$ hombres.

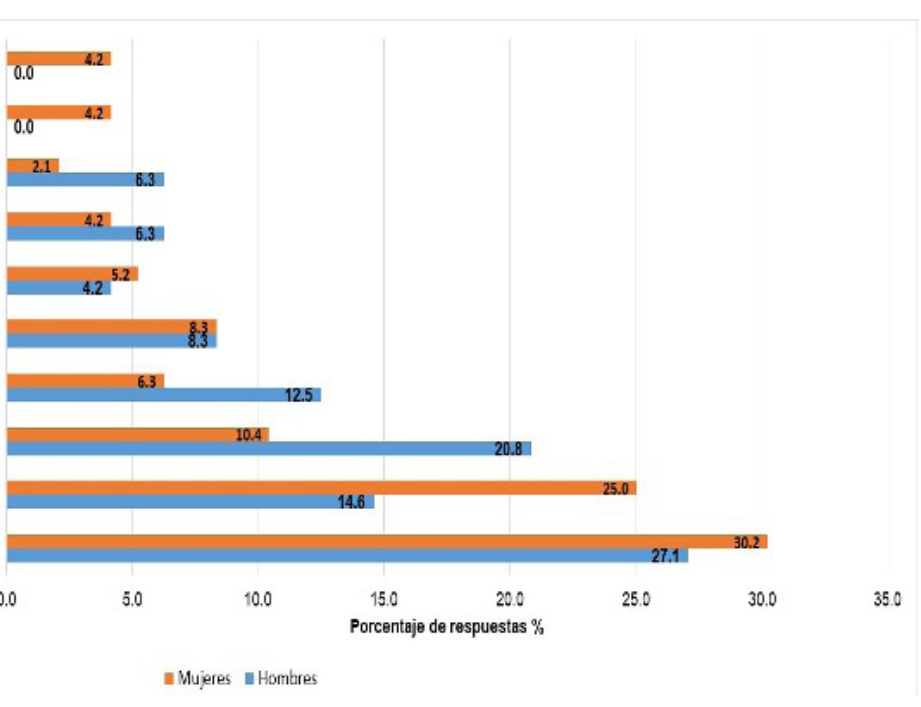


Por otro lado, 14\% dijo haber aprendido nuevas cosas durante la pandemia. En esta categorización se incluyeron opiniones concernientes al aprendizaje de habilidades fuera del ámbito académico o institucional que reportaban los estudiantes. Se recabaron opiniones acerca de alumnos con mayor tiempo y motivación para aprender un nuevo idioma o mejorar su nivel del inglés. De igual manera, hubo diversas opiniones que incluían pasatiempos o desarrollo de habilidades artísticas como la pintura, tocar un instrumento musical, etcétera.

"Tuve tiempo de mejorar mis habilidades en estadística e inglés, mi habilidad en dibujo y hasta empezar a aprender italiano".

"Tiempo para dedicar a actividades relacionadas al arte".

Con $12 \%$ se encuentran las opiniones asociadas al ahorro económico y en tiempo que se invertía en el traslado de los estudiantes de sus hogares a la universidad:

"Evitar viajar en transporte público (promedio de 3 horas diarias)".

"No existe un desgaste en cuestión de invertir dinero y tiempo de traslado".

De igual manera, con 12\% se ubica la categoría que engloba las opiniones concernientes a una mejor organización del tiempo para realizar actividades de su interés:

"Más tiempo para seguir desarrollándome en otras áreas a las cuales no podía dedicarles suficiente atención antes".

"Mayor organización de mis tiempos y actividades".

El resto del porcentaje de las opiniones se divide dentro de categorías con experiencias acerca de capacitaciones en línea o acceso a recursos en línea, con $5 \%$ y con el mismo porcentaje de opinión se encuentran las experiencias referentes a nuevas oportunidades académicas o laborales:

"Posibilidad de hacer más cursos o especializaciones debido a que han tomado el formato virtual".

"Emprender un negocio en el que me ha ido bastante bien."

Por último, con un mismo porcentaje (3\%) de las opiniones realizadas por la muestra de universitarios, se encuentran las categorías relacionadas con un aumento en la capacidad de adaptación, y con el valorar lo que se tiene y un mayor descanso:

"Progreso en mi adaptabilidad para trabajar de manera profesional en diferentes contextos y a distancia."

"Valorar cada momento de vida que tengo".

\section{Experiencias negativas dentro de la pandemia}

Como se muestra en la tabla 3, hubo once clasificaciones temáticas con un total de 127 experiencias negativas expresadas por los participantes incluidos en la investigación. Se puede mostrar el doble de comentarios con experiencias negativas por parte de las mujeres del estudio en ciertos rubros, aunque esto se puede explicar debido a la disparidad en la distribución de la muestra con respecto a hombres y mujeres, con más de la mitad de la muestra mujeres. 
La gráfica 3 representa el porcentaje de opiniones acerca de lo peor que han experimentado los alumnos durante la cuarentena. Como se puede apreciar dentro de las experiencias más negativas que experimentaron los estudiantes encuestados estuvieron principalmente la cancelación de planes académicos, profesionales y personales, lo que conforma $21 \%$ de las experiencias recabadas.

Gráfica 3. Porcentaje de respuestas de "Lo más negativo de la pandemia de covid-19"

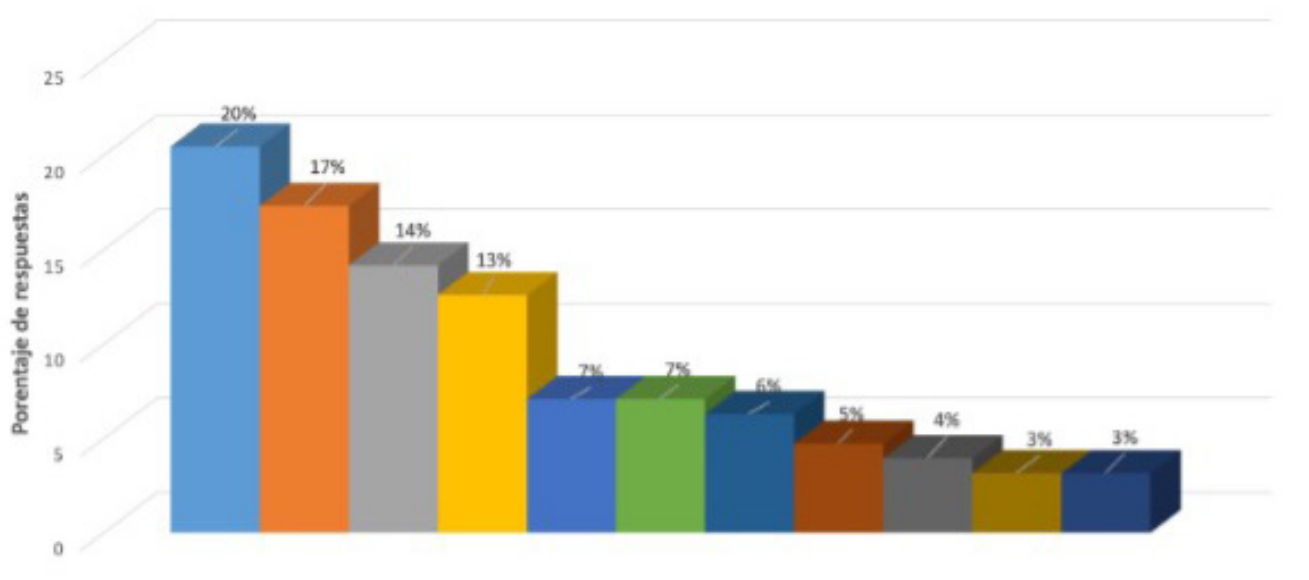

- Cancelar planes personales, académicos y profesionales

- Problemas emocionales

= Sensación de soledad y aislamiento

= Dificultades para adaptarse a la cuarentena

- Problemas económicos o pérdida de empleo

- Conflictos con familiar y pareja

- Fallecimiento de seres queridos

- Sensación de incertidumbre por el futuro

- No poder ver a mis seres queridos

- Contagio de COVID-19

- Miedo al contagio

Diversos comentarios hacían referencia a la cancelación de planes a futuro como estancias académicas o viajes al extranjero, dificultades para la culminación de sus estudios debido al cese temporal en los procesos administrativos en la universidad y postergación de proyectos de investigación que se vieron mermados por la pandemia:

"La pandemia cambió totalmente mis planes para este año, tanto a nivel académico como personal, impactando negativamente en el desarrollo de los estudios de mi tesis, así como en las posibilidades que contemplaba de movilidad a otros países, para un mayor desarrollo personal y profesional".

"Perder un viaje internacional con fines académicos y recreativos, cuya planeación llevaba más de un año y además implicó pérdida de vuelos y hospedaje".

Se pueden identificar experiencias negativas asociadas con los problemas emocionales o psicológicos provocados por la pandemia y por el aislamiento 
social en $17 \%$ de la muestra total. De acuerdo con las experiencias de los participantes del estudio, hubo un incremento en ansiedad, estrés y depresión. Se reportaron también alta labilidad emocional y un incremento de emociones negativas como irritabilidad, preocupación, miedo, tristeza, enojo y frustración. En esta categoría, a simple vista parece que hubo una mayor afectación reportada por las mujeres del estudio en comparación con los hombres, dado que fueron catorce las mujeres y sólo ocho los hombres que compartieron estas experiencias. Sin embargo, al transformar la frecuencia de las experiencias y en términos de porcentaje por sexo, 18.6\% de hombres y $16.6 \%$ de mujeres expresaron estos problemas emocionales, relativizándose esta diferencia. Esto se debe a la disparidad entre número de hombres y mujeres que respondieron a la encuesta o por una diferencia en la expresión de las emociones entre hombres y mujeres.

"Me daba ansiedad a un nivel que me hacía vomitar diariamente una vez al día cuando ya iba mucho tiempo de la pandemia."

"Incertidumbre, estrés, tristeza, enojo, es un cúmulo de emociones que muchas veces no me deja dormir, que me provoca ansiedad, y necesito ayuda para regularme."

Por otro lado, relacionado inherentemente al aislamiento social, los participantes en el estudio reportaron sentimientos de soledad y aislamiento, formándose esta categoría con 14\% del total de opiniones. En su mayoría, esta categoría está constituida por comentarios que hacen alusión a una sensación de soledad y tristeza debido al cese de contacto interpersonal, sobre todo en amistades cercanas y en miembros fuera del núcleo familiar:

"El aislamiento a veces me causa sensación de soledad ya que soy una persona que le gusta el afecto y cercanía social".

"Tener que tener comunicación meramente digital con mis seres queridos".

Otra categoría que es importante resaltar fueron las dificultades para adaptarse a la cuarentena. En esta categoría temática se agruparon experiencias relacionadas con las dificultades que percibieron los alumnos para adaptarse a las nuevas normatividades que exige el distanciamiento social. Comentarios que hacen alusión a la imposibilidad de salir y cambios en la forma de trabajo a distancia conformaron 13\% de la muestra total, por ejemplo:

"Las clases en línea y la escasa interacción con compañeros y docentes, eso dificulta las labores de aprendizaje".

"Mi espacio personal se volvió: trabajo y cuarto de estudio".

Con $7 \%$ se encuentran las vivencias experimentadas con respecto a conflictos familiares o con la pareja. Las temáticas que dentro de esta categoría incluyen desacuerdos y conflictos en la convivencia familiar prolongada por el encierro. Como lo ilustra la gráfica 4, estas experiencias fueron reportadas por $8.3 \%$ del total de mujeres y $4.7 \%$ para los hombres, respectivamente. Esta diferencia puede deberse a la disparidad del número de participantes mujeres (53) y hombres (27). 
"Más roces que pueden llevar a discusiones familiares y de pareja".

"Darme cuenta de las conductas machistas y misóginas que persisten en mi familia, mismas que, aunque he tratado de eliminar, siguen existiendo prejuicios, tabúes y creencias, al cuestionarlas me ha provocado disgustos y peleas familiares".

También con 7\% se observa la categoría que engloba comentarios asociados con problemas económicos o la pérdida de empleo, que fueron reportados entre lo más negativo que provocó la pandemia:

"Lo más negativo que me ha pasado esta cuarentena son las dificultades económicas en mi casa".

"Lo peor de la pandemia es la pérdida de oportunidades laborales y, por ende, severas dificultades económicas con todo lo que éstas acarrean".

Al ser un nuevo virus, el SARS-CoV-2, aparte de las vacunas, no cuenta aún con una cura o tratamiento específico. Como se ha reportado, este nuevo virus puede llegar a ser letal para toda la población, en especial para personas con ciertas comorbilidades (diabetes, obesidad, hipertensión, EPoc y problemas del sistema inmunológico), al igual que llegar a causar mayor afectación a personas mayores de 60 años. En nuestro país, el virus SARs-CoV-2 ha causado la muerte de poco más de 200,000 mexicanos (Secretaría de Salud, 2021). Considerando lo anterior, no es sorpresivo que haya opiniones relacionadas con el fallecimiento de seres queridos dentro de la comunidad estudiantil de la Facultad de Psicología. Éstas constituyeron 5\% de la muestra total.

"Lo más negativo que me ha ocasionado esta pandemia es el fallecimiento de amistades forjadas desde la infancia hasta el ámbito profesional".

"Muerte por Covid de una persona muy importante y la imposibilidad de estar en su velorio por la misma situación de la pandemia".

La sensación de incertidumbre por el futuro ocupó también 5\% de las opiniones de los alumnos. Esto, porque estamos inmersos en el contexto de la pandemia, que está fuera de nuestro control y que depende de diversas variables, como un fin incierto y lejano, o debido a la adaptación a la nueva normalidad, que ha modificado nuestra forma de vida.

"Preocupaciones sobre el futuro respecto a lo que será la vida con el virus y sobre si se restablecerá al menos parcialmente la normalidad de antes."

"Incertidumbre sobre mi futuro y pensamiento de que ya no podréver a mis amigos jamás."

El no poder ver a los seres queridos fue una de las repercusiones negativas reportadas por los estudiantes, con $4 \%$ de las respuestas:

"No poder estar cerca de gente que amo y preocupado de que les pueda pasar algo".

"Aislarme de las personas que estimo cuando de por síya me sentía aislado socialmente".

No obstante, la categoría de miedo al contagio obtuvo un porcentaje relativamente bajo en comparación de las demás (3\%). Esto podría apuntar a que hay un gran temor a ser infectado por el nuevo virus sars-CoV-2 y que, por ende, los encuestados tienden a aislarse de sus seres queridos: 
Gráfica 4. Porcentaje de opiniones, divididas por sexo, de "Lo más negativo de la pandemia de covid-19". Es importante señalar que no hubo una distribución homogénea entre el número de participantes por sexo: $66.3 \%$ mujeres y $33.7 \%$ hombres.
"Miedo a relacionarme con los demás por la posibilidad de contagiarme".

"Ansiedad por aumento de trabajo y por miedo a contraer covID".

El contagio de la enfermedad también resultó ser uno de los efectos más negativos que han tenido los alumnos de la comunidad universitaria (3\%) de la Facultad de Psicología:

"Problemas familiares pues prácticamente toda mi familia paterna se contagió".

"Lo peor ha sido que me contagié de CovID".

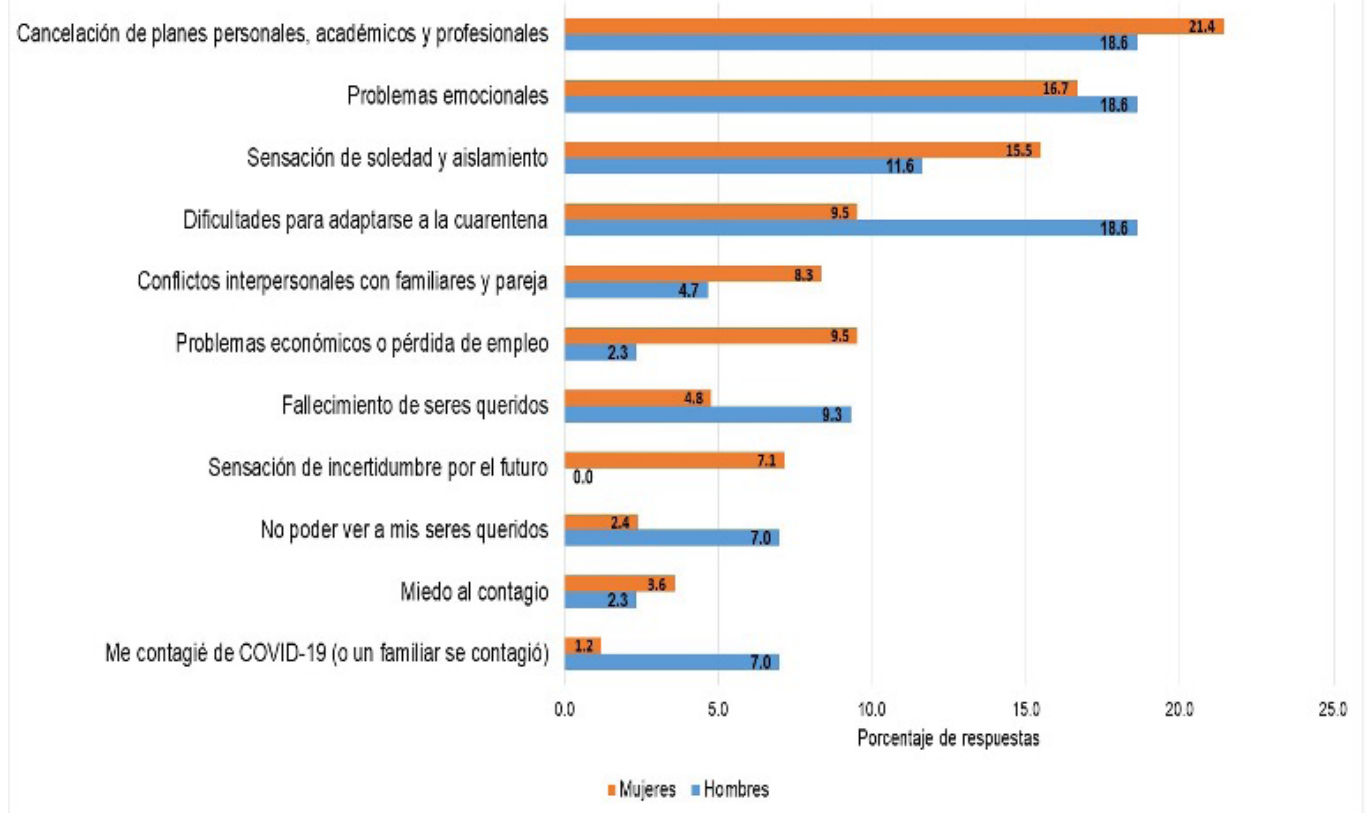

\section{Discusión}

El objetivo de la presente investigación fue explorar las experiencias positivas y negativas que ha traído consigo la pandemia de la covid-19 para la comunidad estudiantil de la Facultad de Psicología de la Universidad Nacional Autónoma de México. Esta pandemia ha planteado grandes retos para los estudiantes universitarios encuestados. La cancelación de planes personales académicos y profesionales fue de las opiniones más reportadas por los alumnos. Esto, a su vez, causó emociones negativas como frustración, enojo e irritabilidad, al igual que exacerbación de problemas psicológicos como ansiedad, estrés y depresión.

Aunado a ello, el aislamiento social trajo consigo un sentimiento de soledad entre los universitarios y se reportaron dificultades para adaptarse a la cuarentena, como la imposibilidad de salir de casa, frecuentar a amistades y familiares o la adaptación a clases en línea y trabajo a distancia. La incertidumbre del progreso y fin de esta emergencia sanitaria repercutió en los alumnos encuestados, reportándose sentimientos de incertidumbre por el futuro. 
Eventos y situaciones causadas directamente por el nuevo virus del SARS-CoV-2 también se reportaron, como el fallecimiento de seres queridos, el miedo al contagio propio o de seres queridos y problemas económicos o pérdida del empleo. Es posible que estas experiencias negativas que se detonaron durante la pandemia estén implicadas en cierta medida con dificultades para tener un buen aprovechamiento en las clases a distancia.

Parte de los hallazgos del presente estudio se corresponden con investigaciones realizadas en otros países. Con respecto a las problemáticas emocionales, suscitadas por la pandemiay reportadas en la presente investigación, hay diversos estudios que hallaron los mismos cambios emocionales en estudiantes universitarios alrededor del mundo. En la investigación realizada por Husky, Kovess-Masfety y Swendsen (2020), con alumnos universitarios franceses, se encontró que el confinamiento por la pandemia de la coviD-19 afectó su salud mental de manera notoria. 60\% de los universitarios en la muestra poblacional de ese estudio mostraron niveles altos de ansiedad y estrés provocados por una situación financiera incierta y preocupación por la salud física de sus seres queridos.

En Bangladesh se encontró que alrededor de 72\% de los estudiantes de distintas universidades de ese país presentaban sintomatología depresiva de leve a moderada, mientras que $87 \%$ tuvo síntomas de ansiedad de leve a moderada a causa de la pandemia (Islam et al., 2020). Los investigadores concluyeron que no sólo el aislamiento social producto de la pandemia acentuó los ya existentes síntomas de ansiedad y depresión en ciertos estudiantes, sino que también afectó su calidad de vida global y su desempeño académico.

Los hallazgos de la presente investigación acerca del miedo al contagio o preocupación por el contagio de sus seres queridos también concuerdan con el estudio de universitarios estadounidenses conducido por Son, Hedge, Smith, Wang y Sasangohar (2020), quienes mencionan que entre las principales afectaciones a los estudiantes se encuentra la preocupación por la salud propia y de los seres queridos, lo cual afecta su concentración y desempeño en actividades académicas.

Otra problemática que se vio reflejada en las respuestas recabadas en la presente investigación fue la de los conflictos familiares o de pareja causados por el aislamiento social y la convivencia prolongada de las personas que habitan en un mismo domicilio. Con base en Zhang (2020), el cohabitar por un mayor tiempo, bajo un contexto de emergencia sanitaria como lo es la pandemia por la covid-19, acentuó problemas dentro del núcleo familiar, o bien, que nuevos estresores como tensiones provocadas por el aislamiento social, cambios radicales en el estilo de vida, desempleo, problemas económicos y contagios de familiares, pudieran generar nuevos conflictos y diferencias entre personas bajo un mismo techo. 
Es importante subrayar que estos conflictos intrafamiliares y de pareja generados por las medidas de distanciamiento social afectan con mayor severidad a las mujeres, sobre todo en Latinoamérica. De acuerdo con onu Mujeres (2021), en la región de América Latina y el Caribe, la pandemia por la covid-19 y el distanciamiento social han afectado desproporcionadamente a las mujeres. Según datos de esta oficina regional de la onu, en Latinoamérica ha aumentado el número de violencia contra las mujeres, ya que en su mayoría éstas viven con su abusador y al respetar las medidas de distanciamiento social se encuentran encerradas, con lo que se dificulta la posibilidad de buscar ayuda y salir de esa situación.

Con este estudio exploratorio también se observó que emergieron aspectos positivos en la vida de los estudiantes a pesar de la pandemia, tales como la posibilidad de mejorar el autocuidado, incluyendo una mejor nutrición, ejercicio, mejores hábitos de sueño y salud mental; oportunidad de aprender nuevas cosas; mejor organización del tiempo; acceso a capacitaciones y cursos en línea gratuitos; nuevas oportunidades laborales; aumento de la capacidad de tolerancia a la incertidumbre y frustración; valorar lo que se tiene; posibilidad de descansar más, y ahorrar tiempo y dinero al evitarse el transporte y los trayectos. Todos los anteriores aspectos también juegan un papel importante en el desempeño académico remoto de la comunidad universitaria.

Al igual que con los negativos, parte de los hallazgos positivos que se encontraron en este estudio exploratorio corresponden con estudios previos relacionados con el tema. En una universidad de Estados Unidos, se reportaron niveles de estrés más bajos en los estudiantes, los investigadores atribuyeron este fenómeno a que al estar confinados se han acercado más a sus familias lo cual les ha brindado apoyo socioemocional (Son, 2020). Esto se puede equiparar con los aspectos positivos señalados en el presente estudio en los cuales se reportó un mayor tiempo con los seres queridos y se relaciona con una sensación de bienestar.

Las experiencias positivas que se encontraron en este estudio referentes a la organización del tiempo coinciden con lo mencionado en el estudio de Miguel (2020), que también encontró que existen alumnos que al estar estudiando desde casa se sienten en paz y ahorran tiempo, lo cual traduce el autor como optimización del tiempo.

Es muy relevante resaltar que algunos participantes reportaron un aumento en su capacidad de adaptarse ante cambios que quedan fuera de su control y poder así tolerar la incertidumbre que está completamente asociada a esta crisis de salud pública. La adaptación es un factor fundamental para sobrellevar esta pandemia. Como lo mencionan Besser, Flett y Zeigler-Hill (2020), una buena capacidad de adaptación en estudiantes universitarios afecta la percepción que tienen acerca de su educación en línea, ya que no sólo se perciben como más autoeficaces para sobrellevar este cambio, sino también mantienen un alto 
nivel de involucramiento y por ende un buen aprendizaje. Como lo explican estos investigadores, una mayor adaptabilidad está asociada con estilos de afrontamiento eficaces y técnicas óptimas de solución de problemas que en su totalidad propician respuestas de resiliencia en la persona. Esto es especialmente importante ya que no sólo la crisis sanitaria está inmersa en incertidumbre en su fin o control, sino que ésta también implica una forzada adaptación a nuevos hábitos, como el uso del cubrebocas, lavado de manos constante y aislamiento social, que protegen al individuo del contagio.

\section{Conclusiones}

Para ofrecer a los alumnos una educación lo más apropiada posible dentro de las condiciones de emergencia sanitaria actual, es importante reflexionar sobre la perspectiva que tienen los estudiantes en cuanto a los aspectos positivos y negativos que ha traído la pandemia por la covid-19 en las distintas áreas de su vida.

Es un hecho que la educación en su modalidad a distancia puede ser complicada por todos los factores negativos con los que deben lidiar los alumnos. Sin embargo, conocer los aspectos positivos que se han generado con la pandemia resulta muy útil, pues de esa forma se conoce qué herramientas se tienen para hacer frente a las dificultades, por ejemplo, el hecho de ahorrar tiempo en el transporte a la universidad repercute de forma positiva para evitar el estrés. La cercanía con las personas con quienes se encuentran confinados los estudiantes les puede ayudar a disminuir su sensación de soledad, o bien, el aumento en conductas de autocuidado puede ayudar a prevenir problemáticas de salud tanto física como psicológica.

Para realizar un traslado adecuado y oportuno a la educación a distancia se recomienda favorecer el nivel de involucramiento en las clases en línea. Como lo señalaron Garris y Fleck (2020), es indispensable promover en esta modalidad que los alumnos estén involucrados. Esto es un doble reto ya que, por sí sola, la educación a distancia tiende a mostrar niveles más bajos de involucramiento en comparación con las clases presenciales; además, con la emergencia sanitaria se acentúa la necesidad de mantener interés entre los estudiantes y el contenido del curso. Esto se puede lograr al utilizar el abanico amplio que ofrecen las TIC, así como al promover la interacción sustancial entre los alumnos y profesores. Como los autores lo señalan en su investigación, altos niveles de interés, disfrute y atención predicen una evaluación positiva de la calidad de la educación y un aprendizaje significativo.

De igual manera, es indispensable tener en mente que el estado emocional de los alumnos, así como las dificultades por las que atraviesan individualmente en las demás esferas de su vida, tienen un impacto en su educación universitaria. Es por esto que es necesario que las instituciones educativas universitarias cuenten con programas y centros de apoyo psicosocial que implementen estrategias 
adaptativas de afrontamiento a la adversidad, con un enfoque específico para la emergencia sanitaria de covID-19. Estas estrategias de afrontamiento y técnicas de solución dotan a la comunidad universitaria de habilidades útiles para afrontar los problemas de forma que aumente su autoeficacia y por ende su resiliencia.

Es importante considerar que la comunidad docente también necesita de este tipo de apoyo psicológico, ya que así podrían maximizar y replicar el uso de competencias socioemocionales de situaciones adversas en sus cursos. De acuerdo con la investigación realizada por Linor, Hadar, Ergas (2020), las competencias socioemocionales propias de los docentes no sólo los protegen contra el burnout o estrés relacionado con la alta carga de trabajo, sino también permiten establecer relaciones empáticas con sus alumnos, las cuales mejoran la calidad de enseñanza durante situaciones adversas. Los hallazgos del estudio reflejaron que, mediante esta relación de confianza y empatía, los profesores pudieron comprender las barreras personales y académicas por las que atravesaban los alumnos durante la pandemia y así fueron capaces de modificar la demanda de trabajo y crear ambientes positivos para potenciar el éxito dentro del aula.

La flexibilidad en la práctica docente es una de las destrezas de mayor utilidad durante estas situaciones de crisis. Entender el contexto por el cual los estudiantes atraviesan en su cotidianidad, y empatizar con las barreras y dificultades que los afectan diferencialmente, permiten a los académicos universitarios modificar la carga de trabajo, adoptar nuevas herramientas pedagógicas y así favorecer el aprendizaje significativo de los estudiantes.

\section{Recomendaciones}

Se recomienda realizar en el futuro investigaciones con metodología cuantitativa, utilizando muestras representativas y proporcionales de toda la comunidad estudiantil universitaria de la UNAM. Esto para poder estudiar cómo afecta la pandemia de la covid-19 a los estudiantes universitarios mexicanos, visualizar posibles diferencias entre carreras, situaciones socioeconómicas y personales, con el fin de empatizar con la situación y poder ofrecer el apoyo que se requiera para maximizar la calidad educativa durante esta crisis sanitaria.

También valdría la pena indagar la perspectiva de los docentes ante este grandísimo reto pedagógico de la migración de la educación universitaria en línea. Es de vital importancia conocer las barreras y aspectos positivos que perciben ante esta emergencia sanitaria, ya que la educación está compuesta por la díada entre alumno y docente.

De igual manera, sería oportuno investigar el rol que tienen las instituciones educativas en el apoyo a los estudiantes y docentes ante esta situación de pandemia. 


\section{Referencias}

- Besser, A., Flett, G. L. y Zeigler-Hill, V. (2020). Adaptability to a Sudden Transition to Online Learning During the CoviD-19 Pandemic: Understanding the Challenges for Students. Scholarship of Teaching and Learning in Psychology. Advance online publication. http://dx.doi.org/10.1037/stl0000198

* Cao, W., Fang, Z., Hou, G., Han, M., Xu, X., Dong, J.y Zheng, J. (2020). The psychological impact of the covID-19 epidemic on college students in China. Psychiatry Research, 287, 112934. http://dx.doi.org/10.1016/j.psychres.2020.112934

- Corbin, J. y Strauss, A. (2015). Basics of Qualitative Research: Techniques and Procedures for Developing Grounded Theory (16th ed.). Sage.

* Dey, I. (2007). Grounding categories. En A. Bryant y K. Charmaz (Eds.), The sAGE Handbook of Grounded Theory (pp. 167-190). SAGE Publications Ltd. https://dx.doi. org/10.4135/9781848607941

- Dzokoto, V., Hicks, T. y Miller, E. (2007). Student lifestyles and emotional well-being at a Historically Black University. Education, 127(4), 511-522. https://core.ac.uk/ download/pdf/56318886.pdf

* Garris, C. P. y Fleck, B. (2020). Student Evaluations of Transitioned-Online Courses During the covID-19 Pandemic. Scholarship of Teaching and Learning in Psychology. Advance online publication. http://dx.doi.org/10.1037/st10000229

* Husky, M. M., Kovess-Masfety, V. y Swendsen, J.D. (2020, octubre). Stress and anxiety among university students in France during Covid-19 mandatory confinement. Comprehensive Psychiatry, 102, 152191. https://doi.org/10.1016/j. comppsych.2020.152191

* Islam, A., Barna, S. D., Raihan, H., Khan, N. A. y Hossain, T. (2020). Depression and anxiety among university students during the CovID-19 pandemic in Bangladesh: A web-based crosssectional survey. PLOS ONE, 15(8), e0238162. https://doi. org/10.1371/journal.pone.0238162

* Hadar, L. L., Ergas, O., Alpert, B. y Ariav, T. (2020). Rethinking teacher education in a vuca world: student teachers' social-emotional competencies during the Covid-19 crisis. European Journal of Teacher Education, 43(4), 573-586, https://doi.org/10.10 80/02619768.2020.1807513

- Marinoni, G., van't Land, H. y Jensen, T. (2020). The impact of covid-19 on Higher Education around the World. IA Global Survey Report. International Association of Universities. https:// www.iau-aiu.net/IMG/pdf/iau_covid19_and_he_survey_report_final_may_2020.pdf

* Miguel, J. (2020). La educación superior en tiempos de pandemia: una visión desde dentro del proceso formativo. Revista Latinoamericana De Estudios Educativos (México). [Recuperado 28 de octubre de 2020] https://www.redalyc. org/jatsRepo/270/27063237017/html/index.html.

- oms (2020) who Director-General's opening remarks at the media briefing on COVID19-11 March 2020 Geneva, Switzerland [recuperado 20 de octubre de 2020]. https://www.who.int/dg/speeches/detail/who-director-general-s-openingremarks-at-the-media-briefing-oncovid-19-11-march-2020. 
oms. (2019). What do we mean by self-care? [recuperado 29 de octubre de 2020] https://www.who.int/reproductivehealth/self-care-interventions/definitions/en/

* onu Mujeres (2021) Prevención de la violencia contra las mujeres frente a Covid-19 en América Latina y el Caribe. [recuperado 26 de marzo de 2021] https://www2.unwomen.org/-/media/field\%20office\%20americas/documentos/ publicaciones/2020/05/es_prevencion\%20de\%20violencia\%20contra\%20las\%20 mujeresbrief\%20espanol.pdf?la=es\&vs=3033

Sanz, I., Sáinz, J. y Capilla, A. (2020). Effects of the coronavirus crisis on education. Madrid: Organization of Ibero-American States for Education, Science and Culture (OEI).

- Secretaria de Salud. (2021). covid-19 - Coronavirus. Coronavirus México. [Recuperado 29 de marzo de 2021] https://coronavirus.gob.mx/covid-19/

- Shaughnessy, J.J., Zechmeister, E.B. y Zechmeister, J.S. (2007) Métodos de Investigación en Psicología. McGraw Hill, 7ma Ed. México.

* Son, C., Hedge, S., Smith, A., Wang, X. y Sasangohar, F. (2020). Effects of covid-19 on College Students' Mental Health in the United States: Interview Survey Study. Journal Of Medical Internet Research, 22(9). https://doi.org/10.2196/21279

- unesco-IESAlC. (2020). Covid-19 and higher education: Today and tomorrow April 9, 20201 Impact analysis, policy responses and recommendations.

Zhang, H. (2020) The Influence of the Ongoing Covid-19 Pandemic on Family

* Violence in China. Journal of Family Violence https://doi.org/10.1007/s10896-02000196-8

\section{Cómo CITAR ESTE ARTículo}

* Melchor Audirac, Andrés, Hernández Zúñiga, Andrea Guadalupe y Sánchez Sosa, Juan José. (2021, mayo-junio). Universitarios mexicanos: lo mejor y lo peor de la pandemia de COVID-19. Revista Digital Universitaria (RDU), 22(3). http://doi. org/10.22201/cuaieed.16076079e.2021.22.3.12 